\title{
Short-Term Effects of Beraprost Sodium on the Markers for Cardiovascular Risk Prediction in Type 2 Diabetic Patients with Microalbuminuria
}

\author{
Yun Mi Choi ${ }^{1}$, Hyuk-Sang Kwon ${ }^{2}$, Kyung Mook Choi ${ }^{3}$, Won-Young Lee ${ }^{4}$, Eun-Gyoung Hong ${ }^{1}$ \\ ${ }^{1}$ Department of Internal Medicine, Hallym University Dongtan Sacred Heart Hospital, Hallym University College of Medicine, \\ Hwaseong; ${ }^{2}$ Department of Internal Medicine, Yeouido St. Mary’s Hospital, College of Medicine, The Catholic University of \\ Korea; ${ }^{3}$ Department of Internal Medicine, Korea University Guro Hospital, Korea University College of Medicine; ${ }^{4}$ Department \\ of Internal Medicine, Kangbuk Samsung Hospital, Sungkyunkwan University School of Medicine, Seoul, Korea
}

Background: To evaluate the changes in cardiovascular risk markers including pulse wave velocity (PWV), microalbuminuria, inflammatory cytokines, and adhesion molecules after treatment with beraprost sodium (BPS) in patients with diabetic nephropathy Methods: This was a multicenter, prospective, randomized, double-blind, placebo-controlled trial. Type 2 diabetes mellitus patients with microalbuminuria were included. The primary endpoints were changes in microalbuminuria in spot urine and PWV after BPS or placebo (PCB) treatment for 24 weeks. The secondary endpoints were changes in clinical and metabolic parameters.

Results: A total of 52 patients completed the 24-week trial. Changes in PWV were not different significantly in the BPS and PCB groups (right, $P=0.16$; left, $P=0.11)$. Changes in microalbuminuria were $14.2 \pm 157.0$ and $34.5 \pm 146.6(\mu \mathrm{g} / \mathrm{mg} \mathrm{Cr}$ ) in the BPS and PCB groups, respectively $(P=0.63)$. Subgroup analysis in the high blood pressure (BP) group (baseline systolic BP $>120 \mathrm{~mm} \mathrm{Hg}$ and diastolic BP $>80 \mathrm{~mm} \mathrm{Hg}$ ), showed that microalbuminuria decreased by -47.6 in the BPS group compared with an increase by $116.4(\mu \mathrm{g} / \mathrm{mg} \mathrm{Cr})$ in the PCB group $(P=0.04)$. Also, in the large waist circumference group $(>95 \mathrm{~cm})$, microalbuminuria decreased significantly in the BPS group $(P=0.04)$.

Conclusion: Short-term treatment of BPS for patients with diabetic nephropathy did not show significant improvement in various cardiovascular risk factors. However, BPS significantly decreased microalbuminuria in study subjects with higher cardiovascular risk such as high BP or large waist circumference.

Keywords: Beraprost; Diabetic nephropathies; Vascular stiffness

\section{INTRODUCTION}

Beraprost sodium (BPS) is a stable, orally active prostacyclin (PGI2) analogue with vasodilatory, antiplatelet, and cytoprotec- tive effects [1]. Through these effects, BPS appears to be an effective agent in the treatment of patients with pulmonary arterial hypertension, Buerger's disease, and arteriosclerosis obliterans [1].
Received: 4 September 2019, Revised: 31 October 2019,

Accepted: 25 November 2019

Corresponding author: Eun-Gyoung Hong

Department of Internal Medicine, Hallym University Dongtan Sacred Heart

Hospital, Hallym University College of Medicine, 7 Keunjaebong-gil, Hwaseong 18450 , Korea

Tel: +82-31-8086-2493, Fax: +82-31-8086-2499, E-mail: hegletter@hallym.or.kr

\section{Copyright $(\odot 2019$ Korean Endocrine Society}

This is an Open Access article distributed under the terms of the Creative Commons Attribution Non-Commercial License (http://creativecommons.org/ licenses/by-nc/4.0/) which permits unrestricted non-commercial use, distribution, and reproduction in any medium, provided the original work is properly cited. 
Also, a previous study in type 2 diabetes mellitus (T2DM) patients showed the anti-atherosclerotic effect of BPS, as assessed by carotid intima-media thickness [2]. Another study recently reported that BPS treatment improved pain in diabetic patients with painful peripheral neuropathy [3].

On the other hand, possible beneficial roles for BPS in diabetic nephropathy have been suggested by some animal studies [46]. Prostaglandins were shown to alleviate angiotensin II-induced constriction of efferent glomerular arterioles and they attenuated glomerular hyperfiltration [7]. The PGI2 analogue, BPS, was shown to reduce urinary albumin excretion in streptozotocin-induced diabetic rats [6], and in human T2DM patients [8].

Based on previous results, we aimed to evaluate the changes in various cardiovascular risk markers including pulse wave velocity (PWV) and microalbuminuria after treatment with BPS in patients with diabetic nephropathy.

\section{METHODS}

\section{Subjects and study design}

This was a multicenter prospective, randomized, double-blind, and placebo-controlled trial. Supplemental Fig. S1 shows the study protocol. T2DM patients aged 19 to 70 years with microalbuminuria, assessed at 1 year from study enrollment by $24-$ hour (30 to $299 \mathrm{mg} /$ day) or spot urine analysis (30 to $299 \mu \mathrm{g} / \mathrm{mg}$ creatinine), were included. There was no change in medication related to diabetes, dyslipidemia, and hypertension for 3 months before enrollment. Exclusion criteria were a history of congestive heart failure or coronary heart disease, a history of any cancer, pregnant or desire to have a baby during the study period, initiation or change in dose of angiotensin converting enzyme inhibitor (ACEi) or angiotensin receptor blocker (ARB) in the previous 3 months, a history of anti-coagulant, anti-platelet, or thrombolytic agents during the previous 3 months, and current use of prostaglandin I 2 medication or endothelin receptor antagonist.

We calculated sample size by PASS 2008 (NCSS, Kaysville, UT, USA) with power (1-beta) $=0.9$ and alpha $=0.05$. Group sample sizes of 24 and 24 were required to detect a difference of -270.0 from the null hypothesis using a two-tailed two-sample $t$ test. Considering possible drop-out and participant distribution between the four study centers, we planned to enroll a total of 80 subjects.

Patients were randomized by permuted block randomization using random numbers and allocated in a 1:1 ratio for the treat- ment (BPS) and placebo control (PCB) groups. After randomization, patients were prescribed study drugs (BPS or PCB) which were visually indistinguishable. The study drugs were prepared and delivered to each center by Astellas Pharma Korea, Inc. (Seoul, Korea). One tablet contained $20 \mu \mathrm{g}$ BPS. Patients were administered 2 tablets three times a day $(120 \mu \mathrm{g} /$ day $)$.

\section{Outcome measures}

The primary endpoints were changes in microalbuminuria in spot urine and PWV after treatment with BPS for 24 weeks. Spot urine microalbuminuria was measured strictly avoiding other affecting factors, such as exercise and drinking. Secondary endpoints included changes in body weight, waist circumference, blood pressure, fasting glucose, $\mathrm{HbA1c}$, lipid profile, serum creatinine, vascular cell adhesion protein 1 (VCAM-1), high-sensitivity C-reactive protein (hs-CRP), and adiponectin level.

Only spot urine microalbuminuria, spot urine creatinine, and cardiovascular surrogate markers including VCAM-1, adiponectin, and hs-CRP were performed in the central laboratory of our institute. Other clinical measurements including PWV measurement and chemical laboratory measures (i.e., fasting glucose, hemoglobin Alc [HbAlc], lipid profile, and serum creatinine) were performed in each local center.

\section{Statistical analysis}

Continuous variables are expressed as mean \pm standard deviation and categorical variables are presented as number (percentage). Continuous variables were compared using the Student's $t$ test or Wilcoxon rank-sum test. Comparisons between each group according to categorical variables were done using a chisquare test or Fisher's exact test (two-tailed). Comparisons between before and after treatment in each group were done using a paired $t$ test or Wilcoxon signed rank test. The $\mathrm{R}$ software package version 2.15.2 (R Foundation for Statistical Computing, Vienna, Austria; www.R-project.org) was used for statistical analysis. All $P$ values were two-tailed, and $P<0.05$ was considered to denote statistical significance.

\section{Ethical approval}

This study was approved by the Institutional Review Boards of Hallym University Dongtan Sacred Heart Hospital (IRB No. 2013-108), the Catholic University Yeouido St. Mary's Hospital, Korea University Guro Hospital, and Sungkyunkwan University Kangbuk Samsung Hospital. Written informed consent was obtained from all patients. During the entire study period, 
patients were followed for any adverse events that were then reported.

\section{RESULTS}

\section{Baseline characteristics}

A total of 61 patients were enrolled and 52 patients completed the 24-week trial. Baseline characteristics are shown in Table 1. Mean age was 53.1 years in the BPS group and 54.6 years in the PCB group. Males comprised $55.6 \%$ and $64.0 \%$ in the BPS and PCB groups, respectively. The PCB group was more obese at baseline. Baseline body mass index (BMI) and waist circumference were $26.8 \mathrm{~kg} / \mathrm{m}^{2}$ versus $28.2 \mathrm{~kg} / \mathrm{m}^{2}$ and $89.7 \mathrm{~cm}$ versus $96.4 \mathrm{~cm}$ in the BPS and PCB groups, respectively $(P=0.03$ and $P=0.03)$. Baseline smoking status, diabetic retinopathy, diabetic peripheral neuropathy, blood pressure, laboratory measures, and concomitant medication status were not different between the two groups (Tables 1,2). At baseline both PWV and microalbu- minuria were not significantly different between the two groups (Table 1).

\section{Primary and secondary outcomes}

Changes in right PWV were $9.0 \pm 162.0 \mathrm{~m} / \mathrm{sec}$ versus $-65.8 \pm$ $209.9 \mathrm{~m} / \mathrm{sec}$ in the BPS and PCB groups, respectively $(P=0.16)$. Changes in left PWV were $35.6 \pm 194.5 \mathrm{~m} / \mathrm{sec}$ versus $-59.2 \pm$ $226.2 \mathrm{~m} / \mathrm{sec}$ in the BPS and PCB groups, respectively $(P=0.11)$. Microalbuminuria changed by $14.2 \pm 157.0$ and $34.5 \pm 146.6 \mu \mathrm{g} /$ $\mathrm{mg} \mathrm{Cr}$ in the BPS and PCB groups, respectively $(P=0.63)$ (Table 3). Among secondary outcomes, systolic blood pressure was significantly decreased in the BPS group. Changes in body weight, waist circumference, blood glucose, cholesterol, and cardiovascular surrogate markers were not different between the two groups (Table 3).

Additionally, clinical and laboratory parameters were compared before and after treatment in each group (Supplemental Tables S1, S2). In the BPS group, waist circumference and $\mathrm{HbA1c}$

\begin{tabular}{|c|c|c|c|}
\hline Characteristic & BPS group $(n=27)$ & PCB group $(n=25)$ & $P$ value \\
\hline Age, yr & $53.1 \pm 9.4$ & $54.6 \pm 12.5$ & 0.61 \\
\hline Male sex & $15(55.6)$ & $16(64.0)$ & 0.74 \\
\hline Smoking & $5(18.5)$ & $7(28.0)$ & 0.63 \\
\hline Diabetes duration, yr & $8.0 \pm 5.7$ & $6.4 \pm 5.7$ & 0.33 \\
\hline Diabetic retinopathy & $8(29.6)$ & $4(16.0)$ & 0.40 \\
\hline Diabetic peripheral neuropathy & $8(29.6)$ & $8(32.0)$ & 1.00 \\
\hline Body weight, $\mathrm{kg}$ & $72.0 \pm 16.4$ & $78.7 \pm 17.5$ & 0.16 \\
\hline BMI, $\mathrm{kg} / \mathrm{m}^{2}$ & $26.8 \pm 5.2$ & $28.2 \pm 4.7$ & 0.03 \\
\hline Waist circumference, $\mathrm{cm}$ & $89.7 \pm 10.0$ & $96.4 \pm 11.4$ & 0.03 \\
\hline Systolic blood pressure, $\mathrm{mm} \mathrm{Hg}$ & $128.4 \pm 10.8$ & $133.6 \pm 11.0$ & 0.09 \\
\hline Diastolic blood pressure, $\mathrm{mm} \mathrm{Hg}$ & $77.9 \pm 8.1$ & $78.3 \pm 8.2$ & 0.84 \\
\hline Fasting blood glucose, $\mathrm{mg} / \mathrm{dL}$ & $143.7 \pm 37.4$ & $148.3 \pm 29.0$ & 0.63 \\
\hline $\mathrm{HbA1c}, \%$ & $7.0 \pm 1.1$ & $7.4 \pm 1.2$ & 0.20 \\
\hline Total cholesterol, mg/dL & $169.4 \pm 35.6$ & $156.4 \pm 34.3$ & 0.18 \\
\hline HDL-C, mg/dL & $42.7 \pm 8.9$ & $44.8 \pm 11.8$ & 0.47 \\
\hline LDL-C, mg/dL & $101.5 \pm 31.1$ & $88.1 \pm 26.2$ & 0.10 \\
\hline Triglycerides, mg/dL & $177.4 \pm 85.4$ & $185.8 \pm 236.8$ & 0.87 \\
\hline Creatinine, $\mathrm{mg} / \mathrm{dL}$ & $0.9 \pm 0.2$ & $1.0 \pm 0.2$ & 0.19 \\
\hline Right PWV, m/sec & $1,498.9 \pm 209.3$ & $1,630.3 \pm 326.1$ & 0.09 \\
\hline Left PWV, m/sec & $1,490.2 \pm 227.2$ & $1,622.0 \pm 303.7$ & 0.09 \\
\hline Microalbuminuria, $\mu \mathrm{g} / \mathrm{mg} \mathrm{Cr}$ & $222.7 \pm 356.7$ & $182.1 \pm 225.3$ & 0.62 \\
\hline
\end{tabular}


Table 2. Comorbidities and Concomitant Medication

\begin{tabular}{lccc}
\hline Variable & $\begin{array}{c}\text { BPS group } \\
(n=27)\end{array}$ & $\begin{array}{c}\text { PCB group } \\
(n=25)\end{array}$ & $P$ value \\
\hline History of hypertension & $13(48.1)$ & $18(72.0)$ & 0.14 \\
Hypertension medication & $11(84.6)$ & $13(72.2)$ & 0.40 \\
RAS inhibitor use & $13(48.1)$ & $16(64.0)$ & 0.38 \\
History of dyslipidemia & $21(77.8)$ & $18(72.0)$ & 0.87 \\
Dyslipidemia medication & $18(85.7)$ & $15(83.3)$ & 1.00 \\
Diabetes medication & & & \\
Insulin & $11(40.7)$ & $5(20.0)$ & 0.19 \\
Metformin & $21(77.8)$ & $23(92.0)$ & 0.30 \\
Thiazolidinedione & $1(3.7)$ & $1(4.0)$ & 1.00 \\
DPP4 inhibitor & $12(44.4)$ & $12(48.0)$ & 1.00 \\
Sulfonylurea & $13(48.1)$ & $15(60.0)$ & 0.56 \\
Meglitinide & $1(3.7)$ & $1(4.0)$ & 1.00 \\
Alpha glucosidase inhibitor & $2(7.4)$ & 0 & 0.51 \\
GLP1-receptor agonist & $1(3.7)$ & 0 & 1.00 \\
\hline
\end{tabular}

Values are expressed as number (\%).

BPS, beraprost sodium; PCB, placebo; RAS, renin angiotensin system; DPP4, dipeptidyl peptidase-4; GLP1, glucagon-like peptide-1.

increased significantly after treatment (waist, $2.8 \pm 5.3 \mathrm{~cm}, P=$ 0.01 ; HbAlc, $0.3 \% \pm 0.6 \%, P=0.02$ ). And in the PCB group, systolic blood pressure was improved $(-7.6 \pm 10.0 \mathrm{~mm} \mathrm{Hg}, P<$ 0.01). In both groups, bilateral PWV and microalbuminuria did not change significantly before and after treatment.

Multiple regression analyses were conducted using several models to adjust for possible confounding factors (baseline and change in waist circumference, $\mathrm{HbAlc}$, and systolic blood pressure during treatment), but the results were not significant (Supplemental Table S3).

\section{Subgroup analysis}

We additionally performed subgroup analysis for blood pressure and waist circumference. In the high blood pressure group (baseline systolic blood pressure $>120 \mathrm{~mm} \mathrm{Hg}$ and baseline diastolic blood pressure $>80 \mathrm{~mm} \mathrm{Hg}$ ), microalbuminuria decreased significantly after BPS treatment compared with the PCB group (Fig. 1A). However, changes in bilateral PWV were not seen (Table 4). Likewise, in the high waist circumference group ( $>95 \mathrm{~cm}$ ), microalbuminuria decreased significantly after BPS treatment but not PWV (Fig. 1B, Table 4). However, this change was not observed in the low blood pressure and small waist circumference groups.

The baseline ACEi/ARB usage group $(n=29)$ showed signifi-
Table 3. Primary and Secondary Endpoints

\begin{tabular}{lccc}
\hline Variable & $\begin{array}{c}\text { BPS group } \\
(n=27)\end{array}$ & $\begin{array}{c}\text { PCB group } \\
(n=25)\end{array}$ & $P$ value \\
\hline Primary endpoint (post-pre) & & & \\
Right PWV, m/sec & $9.0 \pm 162.0$ & $-65.8 \pm 209.9$ & 0.16 \\
Left PWV, m/sec & $35.6 \pm 194.5$ & $-59.2 \pm 226.2$ & 0.11 \\
Microalbuminuria, $\mu$ g/mg Cr & $14.2 \pm 157.0$ & $34.5 \pm 146.6$ & 0.63 \\
Secondary endpoint (post-pre) & & & \\
Body weight, kg & $0.6 \pm 3.0$ & $0.5 \pm 2.3$ & 0.91 \\
Waist circumference, cm & $2.8 \pm 5.3$ & $1.6 \pm 4.7$ & 0.42 \\
Systolic blood pressure, mm Hg & $1.1 \pm 13.8$ & $-7.6 \pm 10.0$ & 0.01 \\
Fasting blood glucose, mg/dL & $-5.9 \pm 28.1$ & $-7.5 \pm 32.6$ & 0.86 \\
HbAlc & $0.3 \pm 0.6$ & $1.5 \pm 6.1$ & 0.33 \\
Total cholesterol, mg/dL & $-5.0 \pm 26.3$ & $4.7 \pm 28.5$ & 0.21 \\
HDL-C, mg/dL & $0.6 \pm 5.5$ & $-1.7 \pm 7.4$ & 0.21 \\
LDL-C, mg/dL & $-6.0 \pm 22.2$ & $-1.2 \pm 15.2$ & 0.37 \\
Triglyceride, mg/dL & $-17.7 \pm 86.2$ & $16.5 \pm 52.0$ & 0.09 \\
Creatinine, mg/dL & $<0.1 \pm 0.1$ & $<0.1 \pm 0.1$ & 0.32 \\
TNF- $\alpha, \mathrm{pg} / \mathrm{mL}$ & $-0.1 \pm 0.8$ & $0.1 \pm 0.4$ & 0.25 \\
Adiponectin, pg/mL & $-0.1 \pm 1.9$ & $0.5 \pm 2.5$ & 0.42 \\
hs-CRP, pg/mL & $<0.1 \pm 0.2$ & $-0.2 \pm 1.2$ & 0.51 \\
VCAM-1, pg/mL & $-6.6 \pm 161.2$ & $18.0 \pm 129.9$ & 0.60 \\
Resistin, pg/mL & $-0.2 \pm 2.2$ & $0.1 \pm 2.6$ & 0.68 \\
\hline & & &
\end{tabular}

Values are expressed as mean \pm standard deviation.

BPS, beraprost sodium; PCB, placebo; PWV, pulse wave velocity; HbA1c, hemoglobin A1c; HDL-C, high density lipoprotein cholesterol; LDL-C, low density lipoprotein cholesterol; TNF- $\alpha$, tumor necrosis factor $\alpha$; hs-CRP, high-sensitivity C-reactive protein; VCAM-1, vascular cell adhesion protein 1 .

cantly decreased bilateral PWV during the study period compared with the non-ACEi/ARB usage group (right PWV change, $-83.0 \pm 189.5$ vs. $43.7 \pm 165.3, P=0.01$; left PWV change, $-64.9 \pm 223.0$ vs. $59.2 \pm 183.1, P=0.03)$. But changes in microalbuminuria did not differ significantly between these two groups (32.2 \pm 182.4 vs. $14.3 \pm 103.4, P=0.66)$. In the ACEi/ARB usage or non-usage groups, there were no differences between primary outcomes. There were only two patients who had been taking anti-platelet agents during the study period. Additionally, there were no changes in primary outcomes according to smoking status.

\section{Safety}

There were three severe adverse events seen, and they were only in the PCB group. Those included acute pancreatitis, acute appendicitis, and unstable angina. Other adverse effects were 

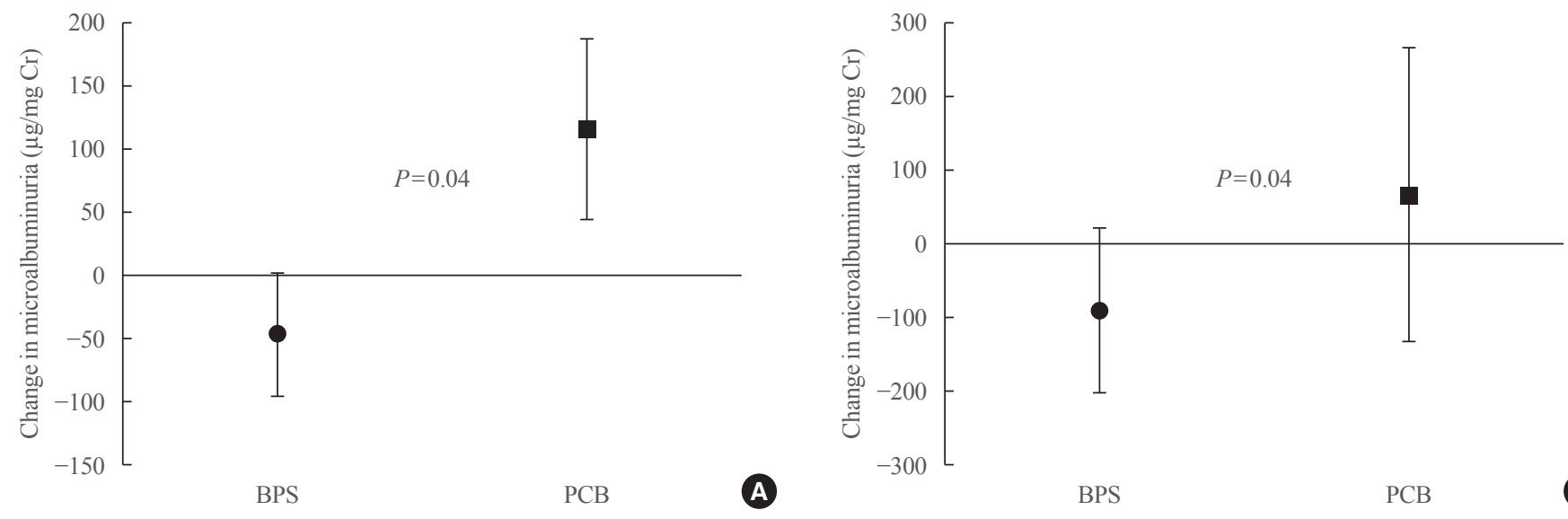

BPS

B

Fig. 1. Changes in microalbuminuria in patients with (A) high blood pressure (BP) (baseline systolic BP >120 mm Hg and diastolic BP $>80 \mathrm{~mm} \mathrm{Hg}$ ), and with (B) large waist circumference (baseline waist circumference $>95 \mathrm{~cm}$ ). BPS, beraprost sodium; PCB, placebo.

\begin{tabular}{|c|c|c|c|}
\hline Variable & BPS group & PCB group & $P$ value \\
\hline \multicolumn{4}{|c|}{ High BP group (baseline SBP > 120 mm Hg and DBP > 80 mm Hg) } \\
\hline Number & 11 & 9 & \\
\hline Changes in right $\mathrm{PWV}, \mathrm{m} / \mathrm{sec}$ & $17.6 \pm 191$ & $-90.7 \pm 185.9$ & 0.22 \\
\hline Changes in left PWV, $\mathrm{m} / \mathrm{sec}$ & $61.9 \pm 230.0$ & $-85.9 \pm 203.4$ & 0.15 \\
\hline \multicolumn{4}{|c|}{ Normal BP group (baseline $\mathrm{SBP} \leq 120 \mathrm{~mm} \mathrm{Hg}$ or $\mathrm{DBP} \leq 80 \mathrm{~mm} \mathrm{Hg}$ ) } \\
\hline Number & 16 & 16 & \\
\hline Changes in right $\mathrm{PWV}, \mathrm{m} / \mathrm{sec}$ & $3.1 \pm 144.6$ & $-51.8 \pm 226.9$ & 0.42 \\
\hline Changes in left PWV, m/sec & $17.6 \pm 171.5$ & $-44.3 \pm 243.1$ & 0.41 \\
\hline \multicolumn{4}{|c|}{ Large WC group (baseline WC >95 cm) } \\
\hline Number & 8 & 12 & \\
\hline Changes in right $\mathrm{PWV}, \mathrm{m} / \mathrm{sec}$ & $-64.4 \pm 123.4$ & $-136.6 \pm 200.6$ & 0.33 \\
\hline Changes in left PWV, m/sec & $9.1 \pm 162.7$ & $-120.7 \pm 219.3$ & 0.15 \\
\hline \multicolumn{4}{|c|}{ Normal WC group (baseline $W C \leq 95 \mathrm{~cm}$ ) } \\
\hline Number & 19 & 13 & \\
\hline Changes in right $\mathrm{PWV}, \mathrm{m} / \mathrm{sec}$ & $39.9 \pm 169.1$ & $-0.5 \pm 203.9$ & 0.56 \\
\hline Changes in left PWV, m/sec & $46.8 \pm 209.5$ & $-2.6 \pm 225.7$ & 0.54 \\
\hline
\end{tabular}

Values are expressed as mean \pm standard deviation.

BPS, beraprost sodium; PCB, placebo; BP, blood pressure; SBP, systolic blood pressure; DBP, diastolic blood pressure; PWV, pulse wave velocity; WC, waist circumference.

not severe and there was no difference in adverse effects between the two main groups (Supplemental Table S4).

\section{DISCUSSION}

PGI2, an endogenous prostaglandin, is the main product of arachidonic acid, which is primarily produced in endothelial cells of all vascular tissues [1]. PGI2 is involved in complex interac- tions between vessel walls, blood flow, and platelet function and it is postulated that it antagonizes platelet aggregation and vasoconstriction caused by endogenous thromboxane A2. The mechanisms of its interactions are likely to involve relaxation of vascular smooth muscle cells, inhibition of platelet aggregation, dispersion of platelet aggregates, inhibition of chemotaxis and cell proliferation, inhibition of production and secretion of endothelin, and cytoprotective effects [1]. 
PWV has been utilized to assess arterial wall stiffness in patients with cardiovascular diseases [9,10]. A larger arterial PWV indicates a greater degree of arterial wall stiffness, consistent with atherosclerotic states. There has been little previous report that evaluated the effect of BPS on arterial stiffness in diabetic patients. One previous study [11] did not show any change in hemodynamic variables such as ankle-brachial index and PWV after BPS treatment in patients with diabetic nephropathy and arteriosclerosis obliterans. There were two previous studies which showed a PWV change after BPS treatment in patients with a history of cerebrovascular accidents $[12,13]$. Both studies demonstrated that 3 months of BPS treatment significantly reduced PWV in patients with cerebral infarction. However, both trials showed significant blood pressure reduction in the BPS group, although it is difficult to differentiate an independent BPS effect on PWV. There also have been reports which showed the vasodilatory effect of PGI2 on cerebral blood flow [14]. However, in this study, we could not show any beneficial effect of BPS on PWV. Generally, PWV is easily affected by blood pressure, obesity, and blood sugar level [15-18]. We assume that the reasons for lack of significant improvement in PWV in our study were: (1) Baseline obesity, particularly waist circumference, was significantly larger in the PCB group, and baseline PWV was slightly higher in the PCB group, though the difference was not significant. On the other hand, systolic blood pressure decreased significantly in the PCB group compared with the BPS group. (2) When comparing variables before and after treatment in each group, waist circumference and HbA1c increased in the BPS group, while systolic blood pressure was decreased in the PCB group.

Diabetic nephropathy is one of the primary diseases of refractory renal failure. It is very important to start treatment in the early stage of the nephropathy. However, any effective drugs other than ACEi have not been reported [8]. The main mechanism is inappropriate dilatation of afferent arterioles, which may induce glomerular hyperfiltration and hypertrophy followed by thickening of the glomerular basement membrane and accumulation of mesangial matrix [19,20]. Increased production of thromboxane A2 in the kidneys may be another factor that aggravates the symptoms of diabetic nephropathy, due to inducing platelet aggregation in the glomeruli [8]. BPS may inhibit platelet-derived growth factor-induced mesangial cell growth [21]. Prostaglandins alleviate angiotensin II-induced constriction of efferent glomerular arterioles in vitro [7,22-24]. Furthermore, they have a very strong inhibitory effect on platelet aggregation, which contradicts the effects of thromboxane A2 [8]. In this study, we could not reproduce the previously reported BPS effect of albuminuria improvement in the general BPS group [8]. One possibility for these results is that metabolic parameters were changed significantly after treatment, as in PWV analysis, although our study was of a relatively short-term design for observing improvement in albuminuria. The improvement of albuminuria in the previous study [8] was observed after 1 year of treatment. Nonetheless, in this study, we performed subgroup analyses and noted a beneficial effect of BPS only in patients with high blood pressure or large waist circumference. Initially, we tried to perform subgroup analysis based on the cutoff value of the standard metabolic syndrome criteria of blood pressure or waist circumference. But overall study subject's blood pressures were well controlled and only one patient exceeded systolic/diastolic blood pressure of 140/90 mm Hg. And 10 subjects (four subjects in BPS groups) exceeded 130/85 mm Hg. For that small portion of subjects it was hard to draw significant results. But blood pressure is very important factor that can affect PWV or microalbuminuria. To reflect blood pressure effect as much as possible, we analyzed based on relatively high blood pressure cutoff value within study subjects. Also in subgroup analysis according to waist circumference, we could not find any significant changes in primary outcomes based on abdominal obesity criteria of Korean Society for Study of Obesity $(90 \mathrm{~cm}$ for men and $85 \mathrm{~cm}$ for women). Then we tried to find out a point of inflection and reanalyzed according to value above a certain degree.

Our current study has several limitations. First, as previously explained, despite the randomization process, waist circumference and BMI were not well controlled between the two groups. Second, during the trial, some variables like blood pressure, $\mathrm{HbAlc}$, and waist circumference changed significantly. Third, combined medications like diabetes medications which can affect primary outcomes were not controlled during treatment. Lastly, for measurement of clinical and laboratory parameters, it might be better if measurements were repeated on a different day. However this study presented more specific groups those who might be more beneficial with BPS treatments.

Further studies with a larger number of patients and a longer observation period are needed to confirm the beneficial effects of BPS on arterial stiffness and microalbuminuria.

\section{CONFLICTS OF INTEREST}

This study was supported by a Research Grant from Astellas Pharma Korea, Inc. (BER-DM 11-003). The funders had no role 
in study design, data collection and analysis, or preparation of the manuscript.

\section{AUTHOR CONTRIBUTION}

Conception or design: E.G.H. Acquisition, analysis, or interpretation of data: Y.M.C., H.S.K., K.M.C., W.Y.L., E.G.H. Drafting the work or revising: Y.M.C., E.G.H. Final approval of the manuscript: E.G.H.

\section{ORCID}

Yun Mi Choi https://orcid.org/0000-0001-8209-874X

Eun-Gyoung Hong https://orcid.org/0000-0003-3390-5706

\section{REFERENCES}

1. Melian EB, Goa KL. Beraprost: a review of its pharmacology and therapeutic efficacy in the treatment of peripheral arterial disease and pulmonary arterial hypertension. Drugs 2002;62:107-33.

2. Goya K, Otsuki M, Xu X, Kasayama S. Effects of the prostaglandin $\mathrm{I} 2$ analogue, beraprost sodium, on vascular cell adhesion molecule-1 expression in human vascular endothelial cells and circulating vascular cell adhesion molecule-1 level in patients with type 2 diabetes mellitus. Metabolism 2003;52:192-8.

3. Shin S, Kim KJ, Chang HJ, Lee BW, Yang WI, Cha BS, et al. The effect of oral prostaglandin analogue on painful diabetic neuropathy: a double-blind, randomized, controlled trial. Diabetes Obes Metab 2013;15:185-8.

4. Guan J, Long L, Chen YQ, Yin Y, Li L, Zhang CX, et al. Effects of beraprost sodium on renal function and inflammatory factors of rats with diabetic nephropathy. Genet Mol Res 2014;13:4154-8.

5. Watanabe M, Nakashima H, Mochizuki S, Abe Y, Ishimura A, Ito K, et al. Amelioration of diabetic nephropathy in OLETF rats by prostaglandin I(2) analog, beraprost sodium. Am J Nephrol 2009;30:1-11.

6. Yamashita T, Shikata K, Matsuda M, Okada S, Ogawa D, Sugimoto H, et al. Beraprost sodium, prostacyclin analogue, attenuates glomerular hyperfiltration and glomerular macrophage infiltration by modulating ecNOS expression in diabetic rats. Diabetes Res Clin Pract 2002;57:149-61.

7. Arima S, Ren Y, Juncos LA, Carretero OA, Ito S. Glomerular prostaglandins modulate vascular reactivity of the down- stream efferent arterioles. Kidney Int 1994;45:650-8.

8. Owada A, Suda S, Hata T. Effect of long-term administration of prostaglandin I(2) in incipient diabetic nephropathy. Nephron 2002;92:788-96.

9. Lehmann ED, Riley WA, Clarkson P, Gosling RG. Non-invasive assessment of cardiovascular disease in diabetes mellitus. Lancet 1997;350 Suppl 1:SI14-9.

10. Amar J, Ruidavets JB, Chamontin B, Drouet L, Ferrieres J. Arterial stiffness and cardiovascular risk factors in a population-based study. J Hypertens 2001;19:381-7.

11. Shima A, Miyamoto M, Kubota Y, Takagi G, Shimizu W. Beraprost sodium protects against diabetic nephropathy in patients with arteriosclerosis obliterans: a prospective, randomized, open-label study. J Nippon Med Sch 2015;82:8491.

12. Nakayama T, Hironaga T, Ishima H, Maruyama T, Masubuchi Y, Kokubun S. The prostacyclin analogue beraprost sodium prevents development of arterial stiffness in elderly patients with cerebral infarction. Prostaglandins Leukot Essent Fatty Acids 2004;70:491-4.

13. Nakayama T, Masubuchi Y, Kawauchi K, Masaki R, Hironaga $\mathrm{T}$, Ishima $\mathrm{H}$, et al. Beneficial effect of beraprost sodium plus telmisartan in the prevention of arterial stiffness development in elderly patients with hypertension and cerebral infarction. Prostaglandins Leukot Essent Fatty Acids 2007;76: 309-14.

14. Chapleau CE, White RP. Effects of prostacyclin on the canine isolated basilar artery. Prostaglandins 1979;17:573-80.

15. Cecelja M, Chowienczyk P. Dissociation of aortic pulse wave velocity with risk factors for cardiovascular disease other than hypertension: a systematic review. Hypertension 2009;54:1328-36.

16. Kim HK, Kim CH, Kim EH, Bae SJ, Choe J, Park JY, et al. Impaired fasting glucose and risk of cardiovascular disease in Korean men and women: the Korean Heart Study. Diabetes Care 2013;36:328-35.

17. Cruickshank K, Riste L, Anderson SG, Wright JS, Dunn G, Gosling RG. Aortic pulse-wave velocity and its relationship to mortality in diabetes and glucose intolerance: an integrated index of vascular function? Circulation 2002;106:208590.

18. Grassi G, Giannattasio C. Obesity and vascular stiffness: when body fat has an adverse impact on arterial dynamics. J Hypertens 2005;23:1789-91.

19. Mauer SM, Steffes MW, Ellis EN, Sutherland DE, Brown DM, Goetz FC. Structural-functional relationships in dia- 
betic nephropathy. J Clin Invest 1984;74:1143-55.

20. Makino H, Yamasaki Y, Haramoto T, Shikata K, Hironaka K, Ota Z, et al. Ultrastructural changes of extracellular matrices in diabetic nephropathy revealed by high resolution scanning and immunoelectron microscopy. Lab Invest 1993;68:45-55.

21. Koh E, Morimoto S, Jiang B, Inoue T, Nabata T, Kitano S, et al. Effects of beraprost sodium, a stable analogue of prostacyclin, on hyperplasia, hypertrophy and glycosaminoglycan synthesis of rat aortic smooth muscle cells. Artery 1993;20: 242-52.

22. Villa E, Rabano A, Ruilope LM, Garcia-Robles R. Effects of cicaprost and fosinopril on the progression of rat diabetic nephropathy. Am J Hypertens 1997;10:202-8.

23. Wang LN, Tang Z, Shou I, Fukui M, Tomino Y. Effects of the PGI2 analog beraprost sodium on glomerular prostanoid synthesis in rats with streptozotocin-induced diabetes. Nephron 1996;73:637-43.

24. Fujita T, Fuke Y, Satomura A, Hidaka M, Ohsawa I, Endo M, et al. PG12 analogue mitigates the progression rate of renal dysfunction improving renal blood flow without glomerular hyperfiltration in patients with chronic renal insufficiency. Prostaglandins Leukot Essent Fatty Acids 2001;65:223-7. 